PACS 81.07.Bc, 61.72.Cc

\title{
On the charge of quodons
}

\author{
J.F.R. Archilla ${ }^{1, \dagger}$, F.M. Russell ${ }^{2}$ \\ †archilla@us.es \\ ${ }^{1}$ Group of Nonlinear Physics, Universidad de Sevilla, ETSI Informática, Avda Reina Mercedes s/n, 41012-Sevilla, Spain \\ ${ }^{2}$ Department of Physics, University of Pretoria, Lynnwood Road, Pretoria 0002, South Africa
}

Muscovite mica is a mineral in which the tracks of charged swift particles, from radioactivity or cosmic rays, can be recorded and made visible by decoration with the mineral magnetite. Also, the tracks of quasi-one-dimensional lattice excitations, called quodons, moving along close-packed directions can be recorded. Most quodon tracks evolve from nuclear recoils following decay of the radioactive isotope ${ }^{40} \mathrm{~K}$ in mica. The recording process is charge sensitive: moving positive charge precipitates black magnetite and moving negative charge leads to clear epidote. Computer modeling of lattice excitations suggest that breathers or solitons can trap a charge. Most of the decays of ${ }^{40} \mathrm{~K}$ leave behind a charge, about $90 \%$ of them positive. So the evolution of most quodons from nuclear recoil events occurs in the presence of a charge. The possible energies and mechanisms of charge transmission in the lattice are analyzed. The outcome of ${ }^{40} \mathrm{~K}$ decay can be $\mathrm{Ca}^{++}, \mathrm{Ar}^{0}, \mathrm{Ar}^{+}$and $\mathrm{Ar}^{++}$. These ions not only have different recoil energy and charge, but also different electron levels and ionization potentials. The first collision of the primary recoiling ion can be inelastic with the transfer of charge to the secondary ion $\mathrm{K}^{+}$. We analyze the possible results taking into account charge, energy and momentum conservation and considering the possible ionization states of the $\mathrm{K}$ atoms, which can be intrinsic localized modes, solitons, kinks or crowdions, travelling charge states and combinations of them.

Keywords: mica muscovite, quodons, crowdions, kinks, breathers, polarons, charge transfer.

\section{Introduction}

Macroscopic tracks of elementary swift charged particles can be seen in a Wilson cloud chamber. Charged particles moving in a supersaturated vapor of water or alcohol in air cause ionization. The resulting ions act as nucleation sites for condensation of the vapor leading to a track that can be seen with the naked eye. A fascinating example is the picture of the first positron ever observed[1], where the curved trajectory of about $20 \mathrm{~cm}$ length of the positron can be seen passing through a $6 \mathrm{~mm}$ lead plate in the presence of a magnetic field (Fig. 1). The sense of curvature in the magnetic field identifies the positive charge of the particle. The track can be seen without the aid of any amplification

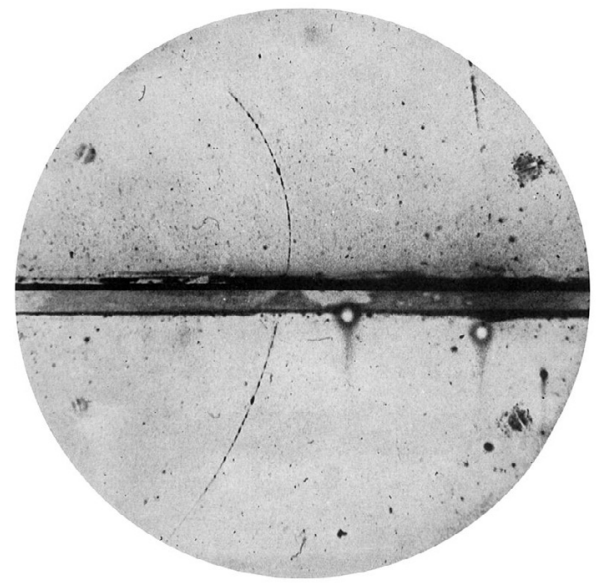

Fig. 1. First positron recorded. The circle diameter is $26 \mathrm{~cm}$. Reproduced from Ref. [1], public domain. process and varies in intensity due to the random ionization process. Also, dots of similar extent can be seen. They may be caused by swift particles moving parallel to the magnetic field, or by spontaneous condensation points.

Similar macroscopic tracks can be observed in layers of the mineral muscovite as shown in Fig. 2. In this case there is a supersaturated solid solution of $\mathrm{Fe}$, which precipitates in the vicinity of a swift particle as it passes by as the mineral magnetite. The initial nano-scale precipitation slowly grows by diffusion and accretion until it eventually stops due to depletion of the iron impurity. The recording and decoration processes are irreversible in the crystals so the tracks persist as the crystals slowly erode to the Earth's surface during millions of years. The duration of local interaction of the positron with the meta-stable mica increases as it slows down, causing increased decoration of the track. The decoration process can continue when the positron is at rest until it is annihilated. This variation of decoration as the positron slows gives rise to the characteristic tadpole shape of the track as seen in Fig. 2. Positrons are produced by $\beta^{+}$decay of the ${ }^{40} \mathrm{~K}$ isotope

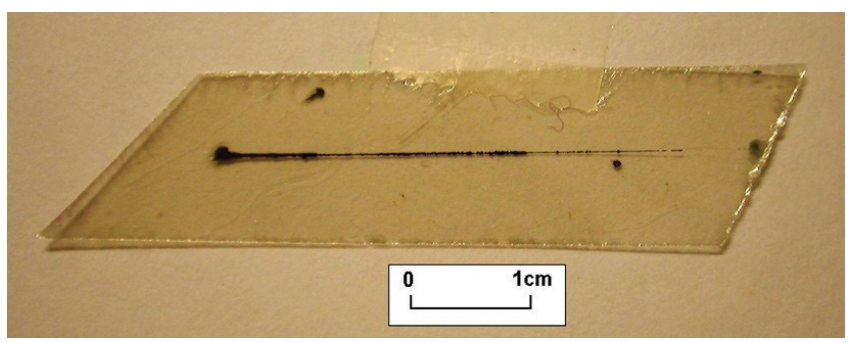

Fig. 2. Positron track in muscovite mica. Reproduced with permission from Ref. [6]. 
present in muscovite with a maximum energy of $0.5 \mathrm{MeV}$. Other swift particles can be recorded, such as anti-muons and protons [2-4]. See Refs. [5, 6] for a recent review. Overlooked during many years has been the fact that only positive charges produce strong decoration with magnetite.

However, only $0.1 \%$ of the tracks have the characteristic kinkiness of a swift charged particle interacting randomly with the atoms in mica. Most of the other observed tracks lie in the hexagonal lattice directions in the $\mathrm{K}^{+}$layer of muscovite in which the ${ }^{40} \mathrm{~K}$ isotope decays. Based on the release of energy and momentum in the decays it was proposed that some kind of lattice excitation was created by the recoil. This quasi-onedimensional particle-like entity was named a quodon[5,7]. Quodons should be highly localized; they travel mostly in one dimension along a chain of atoms in a lattice direction and are able to conserve their energy along macroscopic distances limited only by size of crystal and energy loss by scattering at crystal defects. The longest track measured was $41 \mathrm{~cm}$ or about $10^{9}$ atoms. Their existence was demonstrated experimentally by bombarding one side of a mica crystal with $\alpha$ particles and detecting the ejection of atoms in a lattice direction at the other side of the crystal after propagating more than $10^{7}$ atoms in the crystal[8]. Typical energies for atom ejection in silicates are between $4-8 \mathrm{eV}$, therefore at least some quodons should keep that energy after such a long trajectory.

Recently, a detailed analysis of the decay modes of potassium was performed [9] and it was observed that almost all of the decay modes of ${ }^{40} \mathrm{~K}$ result in a net increase or decrease of electric charge, being the daughters of the ion $\mathrm{K}^{+}$, either $\mathrm{Ar}^{++}, \mathrm{Ar}^{0}, \mathrm{Ca}^{++}$and only around $11 \% \mathrm{Ar}^{+}$with the same charge. In particular, $89 \%$ of the decays is via $\beta^{-}$decay leading to $\mathrm{Ca}^{++}$, giving an excess unit of positive charge. It was noticed that the extent of decoration of the quodon tracks closely matched that of slowly moving positrons[10]. This suggested that quodons leaving a magnetite track might have a unit positive charge. Further, the existence of clear epidote tracks in the opposite direction to a dark magnetite track of a positron suggested the possibility of quodons trapping a negative charge, and thereafter even the possibility of neutral or bare quodons was contemplated[10]. Quodons with different signs of charge probably would have different properties. Trapped charge might also help explain the remarkable stability of quodons both in time and at high temperatures. A unit of charge cannot be divided so if trapped in a quodon it persists until neutralized.

A conspicuous feature of quodons is their ability to create secondary or daughter quodons by scattering at significant lattice defects such as dislocations. This characteristic is illustrated in Fig. 3. It shows a primary quodon with track length $23 \mathrm{~cm}$ that has produced more than 40 secondary quodons lying in different chain directions. A few of the secondary quodons in turn create further secondary quodons. It is seen that the extent of decoration on the primary quodon remains nearly constant despite the repeated loss of energy of the primary quodon in creating the secondary quodons. This shows that the main cause for the decoration is the presence of the trapped charge. Assuming that the initial energy of the primary quodon was the maximum of $42 \mathrm{eV}$ and is dispersed in the 40 -odd secondary quodons then the minimum energy of quodons capable of trapping a charge is of order $1 \mathrm{eV}$ or less if the creation of $2^{\text {nd }}$ order secondary quodons is taken into account.

Since the charge on the primary quodon is conserved a secondary quodon must be created neutral unless it is created in the presence of a free charge. Evidence for this is seen in Fig. 4, which is an enlargement of part of Fig. 3. It is seen that a bare or neutral quodon produces a recorded track with weak decoration. As a neutral quodon propagates it might encounter a charge of either sign. If it traps a positive charge then the decoration becomes comparable to that on the primary. However, it might subsequently encounter a negative charge and revert back to a neutral state, leaving a weak track. This process can repeat, as seen in the Fig. 4. It is noted here that muscovite is an excellent electrical insulator and the decay of ${ }^{40} \mathrm{~K}$ continuously creates randomly distributed charges of both signs.

The objective of this work is to study the possible outcomes of ${ }^{40} \mathrm{~K}$ decay and their consequences for energy and charge transport that may produce the tracks seen in muscovite mica.

\section{Outcomes of ${ }^{40} \mathrm{~K}$ decay.}

The most likely source of energy and charge for quodons is the decay of ${ }^{40} \mathrm{~K}$ into another nucleus due to $\beta$ emission or electron capture. The recoil of the nucleus provides kinetic energy and also charge. The daughter nucleus will have also 40 nucleons, but a different number of protons, an electron

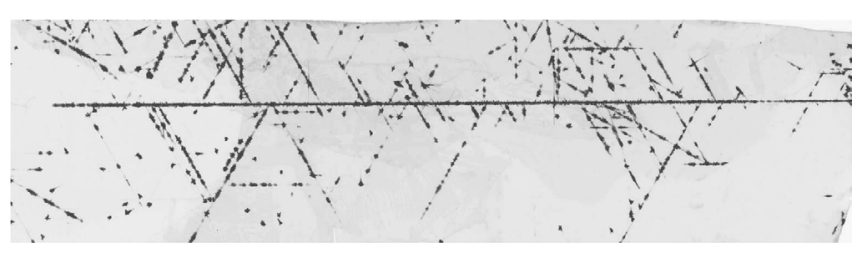

Fig. 3. Photograph of a specimen of mica muscovite of about $20 \mathrm{~cm}$ long. The thick almost horizontal track corresponds to a primary quodon which is scattered by lattice defects creating multiple secondary quodons. They extract energy and momentum from the primary quodon but the width of decoration on the primary quodon remains constant due to presence of a trapped single positive charge. The average energy of secondary quodons is less than $1 / 50$ of the initial energy of the primary quodon.

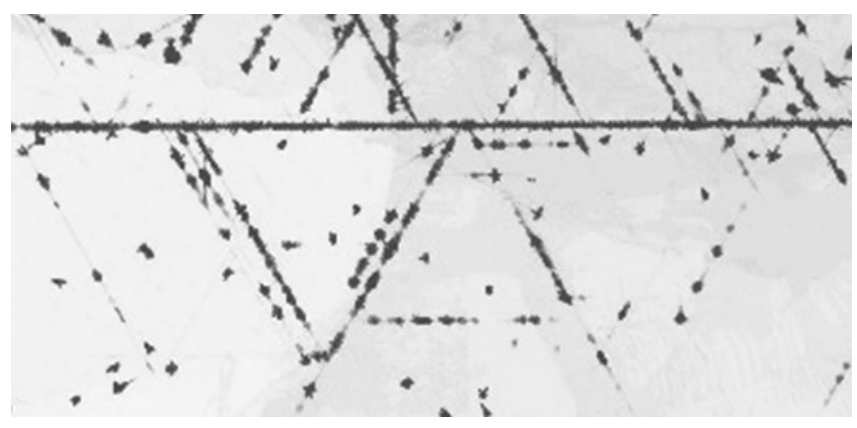

Fig. 4. Amplification of Fig. 3, with a length of about $9 \mathrm{~cm}$. Several interesting features can be easily observed: a) many un-charged secondary quodons with weak decoration trapping single positive charges and producing standard decoration; b) a secondary quodon with double width; c) the intersection of secondary quodons propagating in the same plane. 
or positron may be emitted, a shell electron may be captured by the nucleus in electron capture or emitted from the ion shell in a conversion electron process. The details of the decay modes and their outcome are presented in Table 1 (data from Refs. $[9,11,12])$. In this table the term neutrino is used generically although for $\beta^{-}$emission is actually an electron anti-neutrino. A neutrino is always emitted due to conservation of the electronic number, but in electron capture the captured electron and the nucleus remain together therefore producing two particle events. In EC1 a $44 \mathrm{keV}$ neutrino is emitted and thereafter a $1.46 \mathrm{MeV} \gamma$ or electron with a specific energy bringing about the recoil of the nucleus. In EC2 the pattern is different and the neutrino is more energetic with $1.5 \mathrm{MeV}$ and produces the recoil.

The daughter ions $\mathrm{Ar}^{0}, \mathrm{Ar}^{+}, \mathrm{Ar}^{++}$and $\mathrm{Ca}^{++}$can in principle either recover an electron or lose another one. The simplest measurements of the attachment of an electron to an ion are the ionization potentials. They are given in Table 2 (data from Refs. $[9,13]$. As mica muscovite is neutral and $\mathrm{K}$ is ionized, the lattice has provided the energy for ionization $4.34 \mathrm{eV}$ and a monovalent ion $\mathrm{K}^{+}$at rest is considered the ground state. Therefore the suppression of the positive charge in $\mathrm{K}^{+}$or another ion will need the delivery of $4.34 \mathrm{eV}$ approximately. Through collisions with the daughter of ${ }^{40} \mathrm{~K}$ decay and other factors the ionization state of $\mathrm{K}$ can change. For ease of description we consider the ionization state II as a level for a positive hole with $31.63 \mathrm{eV}$ energy. As a first approximation we suppose that a monovalent positive ion with the same charge have the same energy from the lattice, i.e., $4.34 \mathrm{eV}$. $\mathrm{Ca}^{++}$and specially $\mathrm{Ar}^{++}$will tend to recover their electrons and can therefore provide more energy.

\section{Types of charged excitations.}

The possible types of charged excitations are:

\subsection{A lattice kink or crowdion.}

That is, a traveling compression wave that involves the translation of the particles in one or more lattice units. They are very localized and seen from the outside are equivalent to an interstitial atom traveling at the kink velocity, typically supersonic. For $\mathrm{K}^{+}$ions in the layers of mica muscovite, the interstitial is a potassium ion with a unit of positive charge. Therefore, these lattice kinks, also called crowdions or crowdion waves have positive charge. They can be produced by $\mathrm{K}^{+}$recoil, leaving behind a vacancy or anti-kink. Simulations have been done for 1D models, and at least in some models the kinks do not spread for a long distance $[14,15]$. Supersonic lattice kinks have only a discrete set of velocities and energies for which they do not radiate energy. Only one such value has been found in a 1D model for mica muscovite with realistic parameters with $26 \mathrm{eV}[9,16]$.

It is the hypothesis of this article that ionization processes may occur during collisions. In principle, lattice kinks should have energies below the second ionization state of $\mathrm{K}$, $31.6 \mathrm{eV}$, but actually, due to momentum conservation in an inelastic collision of an atom with energy $E$ with another at rest, $E$ needs to be the double, $62.6 \mathrm{eV}$. This ionization state however can be obtained at the beginning of the quodon, because of the combination of recoil energy from ${ }^{40} \mathrm{~K}$ decay and the ions recovering and an electron and releasing the ionization energy.

Table 1. Decay Table of ${ }^{40} \mathrm{~K}$ decay and its charge result. Data from Refs. [9,11,12].

\begin{tabular}{|c|c|c|c|c|c|}
\hline Decay & $\mathrm{Beta}^{-}$ & EC1 & $\mathrm{EC} 1+\mathrm{CE}^{\star *}$ & $\mathrm{EC} 22^{* * *}$ & Beta $^{+}$ \\
\hline Intensity & $89.25 \%$ & $10.55 \%$ & $0.001 \%$ & $0.2 \%$ & $0.001 \%$ \\
\hline $\mathrm{T}(\mathrm{keV})$ & 1311.07 & 1460 & 1460 & 1504.69 & 483.7 \\
\hline Emitted charged particle & $\mathrm{e}^{-}$ & None & Shell $\mathrm{e}^{-}$ & $\mathrm{e}^{-}$(Auger) & $\mathrm{e}^{+}$ \\
\hline Recoil from & $v+\mathrm{e}^{-}$ & Gamma & Shell $\mathrm{e}^{-}$ & $\mathrm{N}$ & $v+\mathrm{e}^{+}$ \\
\hline Max Recoil (eV) & 42 & $29.2(\mathrm{M})$ & $49.7(\mathrm{M})$ & $31.1(\mathrm{M})$ & 10 \\
\hline Daughter & $\mathrm{Ca}^{++}$ & $\mathrm{Ar}^{+}$ & $\mathrm{Ar}^{++}$ & $\mathrm{Ar}^{++}$ & $\mathrm{Ar}$ \\
\hline $\operatorname{Max} V(\mathrm{Km} / \mathrm{s})$ & 14.4 & $12(\mathrm{M})$ & $15.7(\mathrm{M})$ & $12.2(\mathrm{M})$ & 7 \\
\hline Ionization of daughter $(\mathrm{eV})$ & 50.6 & 27.7 & 40.8 & 40.8 & 15.8 \\
\hline$\Delta \mathrm{q}(\mathrm{e})$ & +1 & 0 & +1 & +1 & -1 \\
\hline \multicolumn{6}{|c|}{$\begin{array}{l}* * \text { Subset of EC1 when the gamma is delivered to a shell electron } \\
\text { (M) Monochromatic } \\
* * * \text { direct decay to Ar ground state, with recoil from } 1504.69 \mathrm{keV} \text { neutrino emission; } 3 \mathrm{keV} \text { Auger } \mathrm{e}^{-} \text {emitted later } \\
\text { Ionization energy of } \mathrm{K}^{+} 31.6\end{array}$} \\
\hline
\end{tabular}

Table 2. Ionization energies (eV) of the atoms involved in ${ }^{40} \mathrm{~K}$ decay $[9,13]$.

\begin{tabular}{|c|c|c|c|c|c|}
\hline & I & II & III & IV & V \\
\hline $\mathrm{Ar}$ & 15.76 & 27.63 & 40.74 & 59.81 & 75.02 \\
\hline $\mathrm{K}$ & 4.34 & 31.63 & 45.81 & 60.91 & 82.66 \\
\hline $\mathrm{Ca}$ & 6.11 & 11.87 & 50.91 & 67.27 & 84.50 \\
\hline
\end{tabular}




\subsection{Moving vacancies.}

The vacancy being the space previously occupied by a positive ion in a neutral crystal has a negative charge. Vacancies have been seen to move in simulations $[17,18]$ but this has not yet been verified in specific models for muscovite.

\subsection{Breathers or intrinsic localizes modes (ILMs) and solitons with a trapped charge.}

They have been described in several simulations in one and two dimensions [19-23]. In principle, breathers or ILMs have an internal vibration, while solitons have not, but in this article they will be identified as the exact nature of the excitation produced by the decay of ${ }^{40} \mathrm{~K}$ is not known. The major difference is if an excitation implies that a $\mathrm{K}^{+}$ion moves a lattice unit to the next lattice site, forcing the ion in that site to move as well and so on. This happens for the crowdion or lattice kink and needs about $26 \mathrm{eV}$ according to Ref. [26]. We will use this value as the energy separating ILMs and solitons for crowdions, although the value should be confirmed by experiments, in order to have an orientation for the possible outcome, but it should not be understood as a precise limit.

\subsection{Ionization states.}

Some or the ${ }^{40} \mathrm{~K}$ recoils have energies which are larger than the second ionization energy of $\mathrm{K}^{+}$, then they may ionize it creating a hole level with energy $31.6 \mathrm{eV}$ above the ground state. This level will be referred here as $|\mathrm{n},+\rangle$. It has only a positive unit of charge due to the negative charge of the medium. It is speculated in this article that the collisions of the ions $\mathrm{K}^{++}$and $\mathrm{K}^{+}$may produce the transfer of the positive hole to the neighboring atom. The probability of it will depend of the matrix element $|<n,+; n+1,0| H|n, 0 ; n+1,+>|$. This matrix element depends on the velocities of the ions $\mathrm{n}$ and $\mathrm{n}+1$ and also in their positions in the external field provided by the layers above and below the potassium one. Most likely there is a combination of positions where the configuration $|\mathrm{n},+1 ; \mathrm{n}, 0\rangle$ is less energetically favorable than $\mid n, 0 ; n+1,+>$ and the transfer of the positive hole takes place. The reason for this is that quodons appear in a layer in a crystal and the lattice should play an essential role.

\subsection{Higher ionization states.}

The ionization of $\mathrm{K}$ to its $3^{\text {rd }}$ level seems unlikely as it would need, according to table 2, $31.63+45.81=77.4 \mathrm{eV}$, which does not seem possible to be provided by ${ }^{40} \mathrm{~K}$ decay and they would entail a higher deformation of the medium and also a strong tendency to recover the electron.

\section{Inelastic collisions}

It is possible that inelastic collisions occur after ${ }^{40} \mathrm{~K}$ decay, particularly $\mathrm{Ar}^{+}$has a strong tendency to recover its electron. The creation of a positive charge will entail an increase of energy of $4.34 \mathrm{eV}$ as this the ionization energy for $\mathrm{K}^{+}$which it is supposed it is delivered by the lattice. Trivially, the equations are:

$$
\begin{gathered}
E_{1}+E_{2}+Q=E_{1}{ }^{\prime}+E_{2}{ }^{\prime} \\
p_{1}+p_{2}=p_{1}{ }^{\prime}+p_{2}{ }^{\prime}
\end{gathered}
$$

with $E=p^{2} / 2 m$.

Or with $E_{2}=0$ and $p_{2}=0$ :

$$
\begin{gathered}
E_{1}+Q=E_{1}{ }^{\prime}+E_{2}{ }^{\prime} \\
p_{1}=p_{1}{ }^{\prime}+p_{2}{ }^{\prime} .
\end{gathered}
$$

$E_{1}$ is given by the recoil of ${ }^{40} \mathrm{~K}$ in the different processes. $Q$ may be positive or negative, depending of the possible charge interchanges and ionization energies. With equal masses and the second atom at rest the condition for Eqs .(2) to have real solutions is

$$
E_{1}>-2 Q
$$

which limits the exothermic processes $(Q<0)$ more than simply the energy balance. In the first collision $m_{1}=40 \mathrm{amu}$, and $m_{2}=39 \mathrm{amu}$, but the change in Eq.(3) is very small.

The first consequence of Eq.(3) is that for the ionization of $\mathrm{K}^{+}$to $\mathrm{K}^{++}$in a $\mathrm{K}^{+}-\mathrm{K}^{+}$collision it would be necessary that the first ion had an energy twice the ionization energy, i.e., $2 \cdot 31.6 \mathrm{eV}=63.2 \mathrm{eV}$. As this energy is not available in any decay, this process is not possible, therefore allowing for more recoil energy than proposed in Refs. $[9,16]$.

Let us analyze the first collisions and their possible outcomes. We will write often the kinetic energy of an ion in $\mathrm{eV}$ in parentheses after the symbol.

A) From $\beta^{+}$decay the result is $\mathrm{Ar}^{0}$ with a continuum of energies from zero to $E_{1}=10 \mathrm{eV} ; \mathrm{Ar}^{0}$ can give 15.7$4.23=11.42$ when recovering the electron, so it is not enough to produce neither $\mathrm{K}^{+} 2^{\text {nd }}$ ionization nor $\mathrm{Ar}^{0} 1^{\text {st }}$ ionization and the collision is elastic.

A.1) $\operatorname{Ar}^{0}(<10)+\mathrm{K}^{+}(0)+Q \rightarrow \operatorname{Ar}^{0}(0)+\mathrm{K}^{+}(<10)$.

This elastic process can produce a breather with maximum $10 \mathrm{eV}$, with no charge attached. The result will be a neutral breather with energies between 0 and $10 \mathrm{eV}$ and a negative charge left behind, because $\operatorname{Ar}^{0}$ cannot compensate the negative charge of the lattice at its site.

$$
\text { A.2) } \mathrm{Ar}^{0}(<10)+\mathrm{K}^{+}(0)+Q \rightarrow \mathrm{Ar}^{+}+\mathrm{K}^{0} \text {; }
$$$$
Q=-15.7+4.53=11.42 \mathrm{eV} \text {. }
$$

As the maximum kinetic energy available is $10 \mathrm{eV}$ this process is not possible according to Eq.(3).

A.3) $\mathrm{Ar}^{0}+\mathrm{K}^{+}+\mathrm{Q} \rightarrow \mathrm{Ar}^{0}+\mathrm{K}^{0}+1 \mathrm{~h}^{+} ; \mathrm{Q}=-4.34 \mathrm{eV}$. This process implies that $E_{2}{ }^{\prime}$ is in $[2.34,4.53] \mathrm{eV}$. Therefore, it is possible a traveling negative charge state $-1 \mathrm{e}$ with energies $[2.34,4.53] \mathrm{eV}$, kept by the elastic process $\mathrm{K}^{0}+\mathrm{K}^{+} \rightarrow \mathrm{K}^{+}+\mathrm{K}^{0}$. It will leave behind a hole $\mathrm{h}^{+}$.

B) From EC1, the result is $\mathrm{Ar}^{+}$with monochromatic energy $E_{1}=29.2 \mathrm{eV}$.

B.1) $\mathrm{Ar}^{+}(29.2)+\mathrm{K}^{+}+Q \rightarrow \mathrm{Ar}^{+}+\mathrm{K}^{+} ; \mathrm{Q}=0$.

The ion $\mathrm{K}^{+}$takes $29.2 \mathrm{eV}$. This process produces a $29.2 \mathrm{eV}$ crowdion $(+1 \mathrm{e})$ with very little excess energy.

B.2) $\mathrm{Ar}^{+}+\mathrm{K}^{+}+Q \rightarrow \mathrm{Ar}^{0}+\mathrm{K}^{++}$;

$Q=15.76-4.34-31.63=20.10 \mathrm{eV}$.

As $E_{1}<-2 Q$, this process is not possible. 
B.3) $\mathrm{Ar}^{+}+\mathrm{K}^{+}+\mathrm{Q} \rightarrow \mathrm{Ar}^{0}+\mathrm{K}^{+}+1 \mathrm{~h}^{+}$;

$Q=15.76-4.34=11.42 \mathrm{eV}$.

This process is possible with $E_{2}{ }^{\prime}=39.9 \mathrm{eV}$ and $E_{1}{ }^{\prime}=0.69 \mathrm{eV}$ backwards. It may produce an energetic crowdion with $39.9 \mathrm{eV}$ and an ILM traveling in opposite direction.

B.4) The process $\mathrm{Ar}^{0}+\mathrm{K}^{+}+\mathrm{Q} \rightarrow \mathrm{Ar}^{+}+\mathrm{K}^{+}$with $\mathrm{Q}=4.34-15.76=-11.42 \mathrm{eV}$ is not possible, therefore the ionization state (-e) backwards cannot occur and the defect charge will remain in Ar.

C) From EC1+CE and EC2. These two processes are monochromatic with $E_{1}=49.7$ and $31.1 \mathrm{eV}$ of $\mathrm{Ar}^{++}$, respectively. $\mathrm{Ar}^{++}$will tend to get and electron releasing $27.63 \mathrm{eV}$ and maybe other one releasing $15.76 \mathrm{eV}$. Of the two, the most interesting is EC2 because the frequency is $0.2 \%$, two hundred times more frequent than $\mathrm{EC} 1+\mathrm{CE}$, with $0.001 \%$.

C.1) $\mathrm{Ar}^{++}+\mathrm{K}^{+}+Q \rightarrow \mathrm{Ar}^{+}+\mathrm{K}^{++} . Q=27.63-31.63=-4 \mathrm{eV}$. This process produces a traveling crowdion $\mathrm{K}^{++}(+2 \mathrm{e})$ with 45.6 or $26.9 \mathrm{eV}$. The $\mathrm{Ar}^{+}$atom is left behind with $E_{1}{ }^{\prime}$ between $0.1-0.2 \mathrm{eV}$ in the same direction.

C.2) $\mathrm{Ar}^{++}+\mathrm{K}^{+}+\mathrm{Q} \rightarrow \mathrm{Ar}^{0}+\mathrm{K}^{++}+1 \mathrm{~h}^{+}$.

$Q=27.63+15.76-31.63-4.34=7.42 \mathrm{eV}$.

This process leaves a traveling crowdion $\mathrm{K}^{++}$with $E_{2}{ }^{\prime}=57.0$ $\mathrm{eV}$ or $38.2 \mathrm{eV}$. $\mathrm{Ar}^{0}$ is left behind with $E_{1}{ }^{\prime}$ between $0.2-0.3 \mathrm{eV}$ backwards.

C.3) $\mathrm{Ar}^{++}+\mathrm{K}^{+}+Q \rightarrow \mathrm{Ar}^{0}+\mathrm{K}^{+++}$.

$Q=27.63+15.76-31.63-45.8-4.34=-38.8 \mathrm{eV}$.

This process is not possible because $E_{1}<-2 Q$.

D) From $\beta^{-}, E_{1}$ is between 0 and $42 \mathrm{eV}$ with $89.25 \%$ of the decays. The possibilities are very similar to the decays with $\mathrm{Ar}^{++}$as outcome, but with smaller 6.11 and $11.87 \mathrm{eV}^{\text {st }}$ and $2^{\text {nd }}$ ionization energies.

D.1) $\mathrm{Ca}^{++}+\mathrm{K}^{+}+\mathrm{Q} \rightarrow \mathrm{Ca}^{++}+\mathrm{K}^{+}$.

Elastic collisions will produce crowdions if $E_{1}>26.2 \mathrm{eV}$ and neutral ILMs if smaller. Due to the mass differences, $\mathrm{Ca}^{++}$ will get a few $\mathrm{meV}$, but basically all the energy is taken by the ion $\mathrm{K}^{+}$. The outcomes can be:

D1.1) If $E_{1}<26.2 \mathrm{eV}$, a neutral ILM with energy $<26.2 \mathrm{eV}$ and a stationary excess charge +1 .

D1.2) If $E_{1}>26.2 \mathrm{eV}$ : a crowdion $(+1)$ with energy in $[26.2,42] \mathrm{eV}$ and a stationary excess charge +1 .

D.2) $\mathrm{Ca}^{++}+\mathrm{K}^{+}+\mathrm{Q} \rightarrow \mathrm{Ca}^{+}+\mathrm{K}^{++}$;

$Q=11.87-31.63=-19.76 \mathrm{eV}$.

If $E_{1}$ is in $[40.1,42] \mathrm{eV}$, then the process is possible with $E_{2}{ }^{\prime}$ in $[10.89,15.6] \mathrm{eV}$ and $E_{1}{ }^{\prime}$ in $[9.5,6.7]^{*} \mathrm{eV}$; That is, there is a positive $\operatorname{ILM}(+1)$ and a neutral ILM travelling in the same direction.

D3) $\mathrm{Ca}^{++}+\mathrm{K}^{+}+\mathrm{Q} \rightarrow \mathrm{Ca}^{0}+\mathrm{K}^{++}+1 \mathrm{~h}^{+}$.

$Q=11.87+6.11-31.63-4.34=-18.00 \mathrm{eV}$.

If $E_{1}$ is in $[36.5,42] \mathrm{eV}$ then the process is possible with $E_{2}^{\prime}$ in $[9.7,19.5] \mathrm{eV}$ and $E_{1}^{\prime}$ in $[8.8,4.5] \mathrm{eV}$. That is, a positive $\operatorname{ILM}(+1 \mathrm{e})$ and a neutral ILM travelling in the same direction. Then:

D4) $\mathrm{Ca}^{++}+\mathrm{K}^{+} \rightarrow \mathrm{Ca}^{+}+\mathrm{K}^{+}+1 \mathrm{~h}^{+} ; \mathrm{Q}=11.87-4.34=7.53 \mathrm{eV}$.

D4.1) For $E_{1}$ in $[0,19.1] \mathrm{eV}, E_{2}{ }^{\prime}$ in $[3.81,26.1] \mathrm{eV}$ and $E_{1}{ }^{\prime}$ in $[3.71,0.5] \mathrm{eV}$ backwards will produce two neutral ILMs in different directions.

D4.2) For $E_{1}$ in $[19.2,42] \mathrm{eV}$ a crowdion $(+1 \mathrm{e})$ with energies $E_{2}^{\prime}$ in $[26.3,49.3]$ and a small ILM with $E_{1}^{\prime}$ in $[0.5$, $0.2] \mathrm{eV}^{\star}$ backwards.

\section{Conclusions}

Muscovite mica has been shown to record the passage of positively charged swift particles as dark tracks decorated with black magnetite. However, most of the observed tracks that lie along the lattice directions are produced by some lattice excitation with positive charge and are called quodons. Also negative quodons may exist and they may leave semi-transparent epidote tracks under appropriate conditions. Evidence for neutral or bare quodons has been found in the scattering of primary quodons. The main source of energy, momentum and charge of the lattice excitations is the recoil and excess charge from the decay of ${ }^{40} \mathrm{~K}$ In this paper we have analyzed the outcomes of the decay which can be $\mathrm{Ar}^{0}, \mathrm{Ar}^{+}, \mathrm{Ar}^{++}$and $\mathrm{Ca}^{++}$, the latter with about $90 \%$ probability. The first collision of the recoiling ion with $\mathrm{K}^{+}$ can be inelastic with the transfer of an electron between ions with the subsequent release or absorption of energy. After the first collision different excitation that transport charge can appear. Among them are positive crowdions, however, the necessity to provide the energy to create an interstitial for a new crowdion in a scattering event makes them unlikely candidates for the tracks corresponding to secondary quodons. Other excitations that can be created are chargetrapping ILMs and solitons; positive and negative traveling charge-states, and also some combinations of them. They must be the explanation of the different types of quodons. We expect that planned simulations and experiment will soon determine each one role.

\section{References}

1. C. D. Anderson. Phys. Rev. 43 (6), 491 (1933).

2. F. M. Russell. Phys. Lett. 25B, 298 (1967).

3. F. M. Russell. Nature 216, 907 (1967).

4. Russel1967c. Nucl. Tracks. Rad. Meas. 15, 41 (1988).

5. F. M. Russell. In Quodons in Mica, J.F. R. Archilla et al, eds., Springer (2015) pp. 3 - 33.

6. F. M. Russell. In Quodons in Mica, J. F. R. Archilla et al, eds., Springer (2015) pp. $474-559$.

7. F. M. Russell, D. R. Collins. Rad. Meas. 25, 67 (1995).

8. F. M. Russell, J.C. Eilbeck. Europhys. Lett. 78, 10004 (2007).

9. J.F. R. Archilla, Y. A. Kosevich, N. Jiménez, V. J. SánchezMorcillo, L.M. García-Raffi, In Quodons in Mica, J. F. R. Archilla et al, eds., Springer (2015) p. 69-96.

10. F. M. Russell. ArXiv: 1505.03185 (2015).

11. X. Mougeot, R. G. Helmer. LNE-LNHB/CEA - Table de Radionucléides, K-40 tables. http://www.nucleide.org (2012).

12. J. Pradler, B. Singh, I. Yavin. Phys. Lett. B 720, 399 (2013).

13. Lide, D. R. (ed.): Handbook of Chemistry and Physics, 90th edn. CRC Press, Boca Raton, Florida, USA (2010). Sec. 10, p. 203.

14. J. Bajars, J. C. Eilbeck, B. Leimkuhler:, Physica D 301, 8 (2015).

15. J. Bajars, J. C. Eilbeck, B. Leimkuhler: In J. F. R. Archilla et al (eds.) Quodons in mica, pp 35 -67. Springer (2015) p35-67. 
16. J. F. R Archilla, Y. A., Kosevich, N. Jiménez, V. J. SánchezMorcillo, L. M. García-Raffi,. Phys. Rev. E 91, 022,912 (2015).

17. J. Cuevas, J., C. Katerji., J.F. R. Archilla, J.C. Eilbeck, F. M. Russell. Phys. Lett. A, 315, 364 (2003).

18. J. Cuevas, J.F. R. Archilla, B. Sánchez-Rey, F. R. Romero. Physica D 216, 115 (2006).

19. Y. Zolotaryuk, J. C. Eilbeck. J. Phys.-Condens. Mat. 10, 4553 (1998).
20. L. Cruzeiro-Hansson, J.C. Eilbeck, J.L. Marin, F. M. Russell, Physica D 142, 101 (2000).

21. D. Hennig, A. Chetverikov, M.G. Velarde, W. Ebeling. Phys. Rev. E 76, 046602 (2007).

22. A.P. Chetverikov, W. Ebeling, M. G. Velarde. Eur. Phys. J.-Spec. Top. 222, 2531 (2013).

23. A.P. Chetverikov, W. Ebeling, and M.G. Velarde. In J.F. R. Archilla et al (eds.) Quodons in mica. Springer (2015) pp. $321-339$. 